
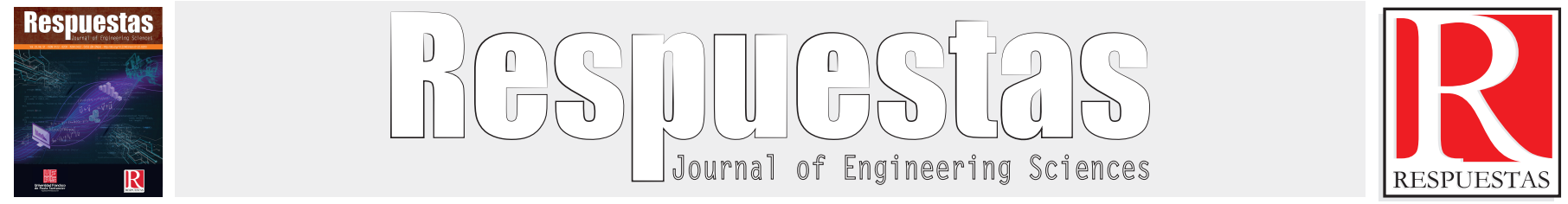

Artículo Original

https://doi.org/10.22463/0122820X.1527

\title{
El programa de las 100 mil viviendas gratis y su impacto en la dinámica social, cultural y ambiental de los beneficiarios de los proyectos del municipio de San José de Cúcuta, periodo 2013-2015
}

The program of 100 million homes, its impact on the social, cultural and environmental dynamics of the beneficiaries of the projects of the municipality of San José de Cúcuta, 2013-2015 period

Luz Karime Coronel-Ruiz.

${ }^{a}$ Magister en Gestión Urbana, luzkarimecr@ufps.edu.co, orcid.org/0000-0003-0331-0568,Universidad Francisco de Paula Santander, Cúcuta, Colombia.

Como citar: L.K. Coronel-Ruiz, "El programa de las 100 mil viviendas gratis y su impacto en la dinámica social, cultural y ambiental de los beneficiarios de los proyectos del municipio de San José de Cúcuta, periodo 2013-2015", Respuestas, vol. 23, no. S1, pp. 81 - 85, 2018. https://doi.org/10.22463/0122820X.1527

Recibido: Febrero 05, 2018; Aprobado: Julio 26, 2018.

\begin{tabular}{ll}
\hline RESUMEN \\
\hline Palabras Clave: & El concepto de vivienda digna, consagrado en el artículo 51 de la constitución política colombiana, establece que todos los \\
Dinámica Ambiental & colombianos tenemos como derecho fundamental el acceso a una vivienda en condiciones que garanticen su disfrute y confort \\
Dinámica Cultural & prime que genera un pilar de desarrollo estratégico en el plan de gobierno del expresidente Juan Manuel Santos durante su \\
Dinámica Social & mandato. Con la adopción del programa nacional de las "Cien Mil Viviendas Gratis para los más Vulnerables", el gobierno \\
Impacto & promueve la adjudicación de viviendas con carácter de gratuidad para aquellas comunidades sisbenizadas de los estratos 1 y 2, \\
Vivienda & victimas del posconflicto y damnificados, que debido a su frágil condición socio-económica, no podían acceder a otros métodos \\
& de adquisición de vivienda formal en los diferentes puntos del suelo nacional. En concordancia, la aplicación de esta nueva \\
& política pública, trajo consigo nuevas dinámicas territoriales durante su proceso de implementación, dinámicas, que aún siguen \\
& manifestándose en los proyectos de vivienda que fueron ejecutados, como es el caso de los proyectos denominados: \\
& Cormoranes, San Fernando del Rodeo, Ciudad Rodeo y Los Estoraques, del municipio de San José de Cúcuta. El presente \\
estudio de caso de investigación, esta direccionado a conocer de manera más clara el impacto que ha tenido mencionado & programa nacional, sobre sus beneficiarios directos en estos proyectos de vivienda en el municipio de Cúcuta y las dinámicas \\
sociales, culturales y ambientales subyacentes.
\end{tabular}

\section{ABSTRACT}

Keywords:

Environmental Dynamics

Cultural Dynamics

Social Dynamics

Impact

Housing
The concept of decent housing, enshrined in Article 51 of the Colombian political constitution, establishes that all Colombians have as a fundamental right the access to housing in conditions that guarantee their enjoyment and comfort, a framework that generates a pillar of strategic development in the government plan of former President Juan Manuel Santos during his first presidential term in the years 2010 - 2014 and that he takes up more strongly in the subsequent years of his second term. With the adoption of the national program of the 'One Hundred Thousand Free Housing for the Most Vulnerable', the government promotes the awarding of free housing for those communities located in strata 1 and 2, victims of the post-conflict and victims, which due to their fragile socio-economic condition, they could not access other methods of acquiring formal housing in the different points of the national soil. In agreement, the application of this new public policy, brought with it new territorial dynamics during its implementation process, dynamics, which are still manifesting in the housing projects that were executed, as is the case of the projects called Cormoranes, San Fernando del Rodeo, Ciudad Rodeo and Los Estoraques, municipality of San José de Cúcuta. The present case study of research is aimed at knowing more clearly the impact that the national program has had on its direct beneficiaries in these housing projects in the municipality of Cúcuta and the underlying social, cultural and environmental dynamics.

\section{Introducción}

El concepto de vivienda digna, consagrado en el artículo 51 de la constitución política colombiana, establece que todos los colombianos tenemos como derecho fundamental el acceso a una vivienda en condiciones que garanticen su disfrute y confort [1]. Para el año 2011, la demanda de vivienda en Colombia se expresa en 3.828.055 hogares, de ellos: 2.520.298 por déficit cualitativo, y 1.307.757 por déficit cuantitativo [2], sumados a los hogares afectados por la ola invernal, cifra que asciende a 2.350.207 [3].

En reflexión histórica, las políticas de vivienda colombianas han estado enfocadas en la oferta de subsidios e incentivos parciales con el objeto de dotar a las personas de menor ingreso y jefes de hogar de la capacidad de compra de un bien inmueble.

Sin embargo, la capacidad de "compra" no es una condición que opere como común denominador de los estratos 1 y 2 del país y mucho menos en aquellos afectados por el conflicto armado interno o el efecto de la ola invernal que afecto el país durante el fenómeno de la Niña de los años 2010 y 2011.

Como consecuencia, la informalidad en las ciudades se ve disparada a través del engrosamiento de los cinturones de miseria, que en la última década fueron y son el reflejo de los altos niveles de pobreza, segregación social y marginalidad urbana de estas comunidades.

*Autor de correspondencia. 
En respuesta a estas realidades, el programa nacional de gobierno 2014-2018 liderado por el expresidente Santos, realiza un trabajo de reingeniería del sistema de subsidios de a través de la sanción de la norma nacional 1537 del año 2012, que abre la puerta al programa de vivienda de las "100 mil viviendas gratis".

En referencia a lo anterior, las administraciones locales de todo el territorio nacional, comienzan los procesos de postulación a esta variación de subsidios en especie, de manera autónoma o con asociaciones público-privadas que les permitiera cumplir con los requisitos técnicos, jurídicos y financieros para la efectiva asignación de cupos dentro del programa, lo cual generó durante los años 2012 a 2014 la contratación para la construcción de 100 mil viviendas gratis en 281 proyectos, de 29 departamentos del país [4].

El municipio de San José de Cúcuta, capital del departamento Norte de Santander, toma parte activa en mencionado proceso y recibe para el año 2013, un número de 3.125 cupos, distribuidos en cuatro proyectos de vivienda sobre el sector noroccidental de la ciudad, denominados: Ciudadela los Estoraques, Ciudad Rodeo, San Fernando del Rodeo y Cormoranes.

La capital fronteriza del oriente colombiano, está localizada en el eje de las migraciones más penosas que se han sufrido en el país durante los últimos años. $\mathrm{Su}$ vecindad con el conflictivo país venezolano, la proximidad con la región del Catatumbo considerada hasta hoy en día como zona de guerrillas, ha traído consigo movimientos poblacionales a gran escala hacia la ciudad, generando la aparición de alrededor de 145 asentamientos informales para el año 2013 [5], [6].

Sumado a esto, la ola invernal que afectó el país durante los años 2010 y 2011, dejó en el municipio más de 1.000 damnificados [3].

El déficit habitacional del municipio de San José de Cúcuta para esos años ascendía a un total es de 41.704 hogares, así: un déficit cuantitativo de 18.597 unidades y un déficit cualitativo es de 23.107 viviendas [7], cuya mayor concentración se encontraba localizada en la cabecera municipal.

Ahora bien, con la ventana de oportunidad presentada por el programa de "las 100.00" para cubrir la necesidad de vivienda del municipio, se realiza la postulación de cuatro (4) proyectos denominados así: San Fernando del Rodeo, Ciudad Rodeo, Los Estoraques y Cormoranes, en asociación con constructores privados y la administración municipal, habilitando el suelo urbanizable y planteando el desarrollo de un número significativo de unidades habitacionales [8].

Mencionados proyectos actualmente ejecutados, se encuentran localizados en la zona de expansión del municipio, como alternativa a la falta de suelo urbanizable dentro del perímetro urbano de la ciudad (Ver figura 1), aprovechando la articulación a nivel de movilidad proporcionada por anillo vial occidental.
UBICACIÓN GENERAL

PROYECTOS DE VIVIENDA GRATIS

Programa $\mathbf{1 0 0}$ mil Viviendas Gobierno Nacional

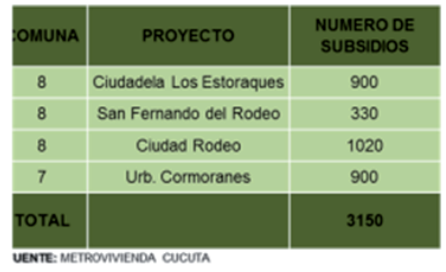

1. Proyecto cormoranes

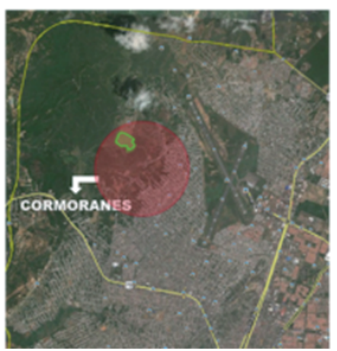

2. Proyecto. Ciudadela los Estoraques
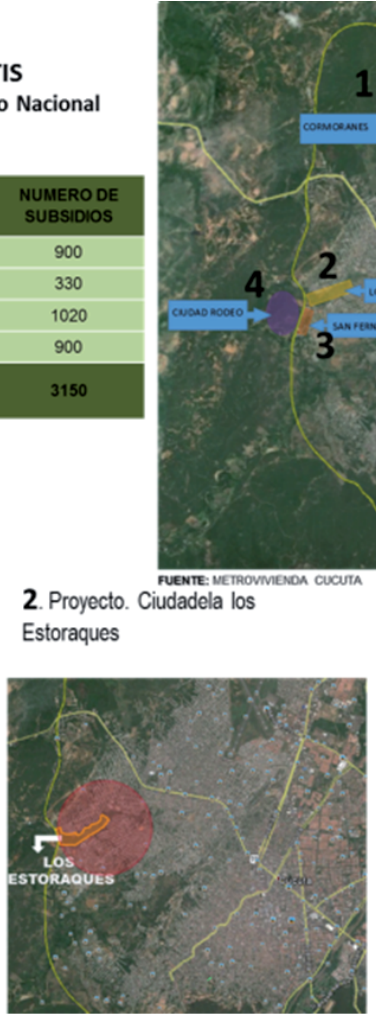

3. Proyecto. San Fernando de Rodeo y 4. Ciudad Rodeo

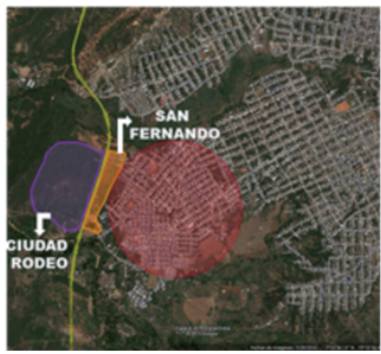

Figura 1. Ubicación general de los proyectos de viviendas gratis Fuente: Ministerio de Vivienda, Ciudad y Territorio 2012 
Sin embargo, hablar de zonas de expansión urbana, es entender que, si bien el suelo está preparado para una futura urbanización y existe un planeamiento mínimo en cuestiones como infraestructura vial y de servicios públicos básicos como: luz, acueducto y alcantarillado, no necesariamente cumple con las condiciones en materia educativa, cultural, económica, recreativa y de salud, que permitan generar un ambiente de bienestar integral para las comunidades asentadas, que como en el caso de San José de Cúcuta, se hicieron evidentes desde la etapa inicial posterior a la entrega de los apartamentos y casas en los proyectos de vivienda de las 100.000; en donde el impacto de esta "nueva vida" genero cambios drásticos en las dinámicas culturales, ambientales y sociales de los beneficiarios.

\section{Materiales y métodos}

Mientras los procesos de postulación de tierras para construcción de proyectos de vivienda gratis se gestionaban desde las administraciones locales, el Departamento Para La Prosperidad Social (DPS) se encargaba de la caracterización y postulación de posibles candidatos a la consecución de subsidio de vivienda en especie con carácter de gratuidad [6].

Víctimas del posconflicto, damnificados por la ola invernal 2010-2011, mujeres cabeza de hogar, población Sisben 1 y 2, fueron evaluados por agentes sociales del DPS para conformar la bolsa de beneficiarios susceptibles a incorporarse en el programa de las 100.000; lo cual arrojaría para el municipio de San José de Cúcuta: 3.150 subsidios efectivos [9].

Un rango heterogéneo de población que de un día para otro se vio en la obligatoriedad de convivir en un espacio de suelo sin la infraestructura requerida para cubrir la demanda, carente de equipamiento que permitiese cumplir con las expectativas mínimas de calidad de vida como: educación, empleo, salud, cultura y recreación.

Producto de esta nueva realidad: la inseguridad, violencia, pandillas, micro-tráfico, intolerancia, prostitución, resistencia al cambio, falta de sentido de pertenencia y apropiación del espacio vital, hace indiscutible la inexistencia de garantías a los núcleos familiares reasentados en las zonas de influencia del programa $[10],[2,[1]$.

Fue entonces cuando se hizo evidente la inexistencia de un marco estratégico claro que permitiese vincular realmente a la familia con su entorno. Carencias como la falta de: transporte público, centros de salud cercanos, estación de policía, centros de desarrollo infantil, centros de enseñanza para la vida productiva, básica y media por nombrar algunas causales, son manifiestas de la falta presencia institucional y acompañamiento tras la entrega de las viviendas, lo cual, desencadenó el caos a nivel general, dando muestra fehaciente de la necesidad de re-estruc- turación del enfoque del programa y su impacto pre y post en la forma de abordaje de la población target.

A partir de mencionados antecedentes, el estudio de caso toma forma, entendiéndose como de vital importancia realizar una profundización sobre los aspectos más relevantes en la coyuntura presentada por las dinámicas ambientales en referencia a: entorno físico y ecológico relacionado con infraestructura y equipamientos; cultura relacionada con: costumbres e identidad; y sociedad relacionada con: sostenibilidad económica y conceptos de vecindad.

Lo anterior, como apuesta a la generación de estrategias que permitan proponer acciones en programas futuros, en lo referente al entendimiento de la repercusión de los mismos sobre las comunidades objeto de intervención y la reducción del impacto negativo.

Durante el proceso investigativo, se elabora un instrumento metodológico como el fin de determinar si el impacto del programa de las 100.000 en los proyectos de vivienda gratis del municipio de Cúcuta, tuvo el resultado deseado en las dinámicas: cultural, social y ambiental.

Para tal fin, se formularon cincuenta y nueve preguntas, estructuradas a partir de referentes como: la ficha de caracterización a nivel familiar de la Organización Panamericana de la Salud y Protección Social, el Anexo 6b de herramientas para la vivienda familiar y el trabajo de investigación titulado: "Consideraciones en torno a la calidad de vida y el paisaje cultural del corregimiento de la Parada, Norte de Santander, que permitieron dilucidar a partir de una toma de muestra estratificada simple a las comunidades de los cuatro proyectos de vivienda, las realidad del impacto del programa [11].

\section{Resultados y Análisis}

En concordancia al desarrollo de la investigación, se establecieron los siguientes resultados producto de la toma de muestra descrita anteriormente:

a) Dinámica Ambiental: calificada como altamente vulnerable, generando un porcentaje de insatisfacción con un $81,4 \%$, porcentaje basado en: inexistencia de equipamiento urbano que garantice cobertura de las necesidades de las familias, escasez de movilidad y transporte incentivando la piratería en la zona y falta de cobertura a nivel educativo y de salud.

b) Dinámica social: calificada como altamente vulnerable generando un porcentaje de insatisfacción con un 76,2\%, porcentaje basado en: insuficiencia de oportunidades laborales dentro del área de influencia y falta de acceso a proyectos de emprendimiento o educación terciaria. 
c) Dinámica Cultural: con un $69,1 \%$ de insatisfacción, deja en evidencia que los conflictos de identidad y territorio están en un punto crítico, en donde los problemas de convivencia, irrespeto por la idiosincrasia y costumbres del otro no han sido vencidos.

En conclusión, se establece como necesario, construir un plan de acciones dirigidas al mejoramiento y corrección de estas situaciones, de manera coherente con la población y sus realidades, que pueda efectivamente lograr un cambio.

Por lo tanto, que se han planteado 3 grandes estrategias que permitirán el desarrollo de acciones específicas guiadas a combatir el impacto negativo en las dinámicas social, ambiental y cultural de los beneficiarios de los proyectos de vivienda gratis del municipio de San José de Cúcuta:

Fortalecimiento Del Espacio Público Tipo 1 Y Tipo 2: Aplicación de las pólizas de estabilidad de los proyectos las cuales tienen un periodo de (5) años para los proyectos de Vivienda, aseguramiento de recursos por parte del municipio para la terminación de la infraestructura necesaria para los proyectos con apoyo en la inversión por parte del gobierno, continuando la política de vivienda y formulación del plan vial de movilidad sostenible.

Estructuración De La Cadena Integral De Seguridad Y Convivencia Ciudadana: Programa de generación de empleo a la población vulnerable o de escasos recursos económicos, presencia de la fuerza pública en el sector, convenios tripartita (municipio, empresa de servicios públicos y las familias para refinanciación y planes de pago), participación del Municipio con programas a mujeres y madres cabeza de familia y decreto No 0847 del 25 de abril del 2013 [12].

Re Significación Cultural proyectos Vivienda Gratis: Espacios de sensibilización y recuperación de la identidad y acompañamiento psicosocial.

\section{Conclusiones}

A partir del estudio de caso realizado, se concluye que el concepto de "vivienda digna" parte principalmente de su imprecisa interpretación, ya que, si bien el espíritu del programa trazado por el gobierno nacional es loable y plantea un impacto económico a gran escala sobre las familias dándoles un bien inmueble patrimonio, no se compromete con el factor humano, priorizando los componentes técnicos y jurídicos, marginando lo social, lo cultural y lo ambiental a segundo plano.

Así también, se determina que la ejecución de la política Nacional de Vivienda de las "100.000" del periodo presidencial Santos, en el marco de la crisis económica y de orden público que atravesaba y atraviesa el país, puede decirse que no ha contrarrestado los altos índices de pobreza en los sectores y comunidades de impacto del programa, en virtud de un proceso que al no involucrar de manera real a quienes son sus usuarios, hace que la solución propuesta, solo rasgue la superficie del problema.

Por otro lado, es de suma prioridad establecer un plan maestro de aplicación en programas similares futuros que no solo contenga el factor técnico, financiero y jurídico del proyecto, sino también el factor humano como eje transversal y director de los procesos de formulación y estructuración.

Como conclusión final, y a manera de aporte general a estas iniciativas gubernamentales, es importante resaltar la necesidad de crear las condiciones propicias previas a la implementación de proyectos relacionados [13].

"La vivienda y sobre todo cuando está dirigida poblaciones vulnerables, deberá gozar de un medio ambiente físico, social y cultural, sano y sostenible".

\section{Referencias}

[1] Asamblea Constituyente. Constitución Política Colombiana, Bogotá: Palacio de Gobierno; 1991.

[2] Alcaldía de San José de Cúcuta. Plan de Desarrollo Municipal 2012 - 2015, Cúcuta: Alcaldia de San Jose de Cúcuta; 2012.

[3] Colombia Humanitaria. Reporte Ola Invernal. Alta Consejería - Presidencia de la Republica de Colombia, Bogota: La Presidencia; 2012.

[4] Ministerio de Vivienda, Ciudad y Territorio. Informe Rendición de Cuentas 2014. Bogota: Presidencia de la República de Colombia; 2014.

[5] Departamento Nacional de Planeación. Boletín de Divulgación Económica, Bogotá: DNP; 2010.

[6] Departamento para la Prosperidad Social. Prosperidad Social. [Internet] 2012 [Citado el 23 de Agosto de 2018]. Disponible en: http://www.dps.gov.co

[7] Camacol Regional Norte de Santander. Informe Actividad Edificadora 2012. Cúcuta: Caracol; 2012.

[8] Metrovivienda Cúcuta. Pagina principal. [Internet] 2013 [Citado el 10 Enero 2017]. Disponible en: http://www.metroviviendacucuta.gov.co

[9] Findeter. Pagina principal. [Internet] 2013 [Citado el 02 
Enero 2017]. Disponible en: http://www.findeter.gov.co

[10] Giraldo F. Las casas "gratis" del gobierno no son como las pintan.[Internet] 2014 [Citado el 02 Enero 2017]. Disponible en: http://www.razonpublica.com(I).html

[11] Durán M, Emilse D. Laboratorio de enseñanza de energías limpias: el caso de la Universidad Simón Bolívar y su impacto en la comunidad Camurí Grande - Anare (Venezuela). Rev Resp, 2016. 21(1), 16-27, 2016.

[12] Ministerio de Vivienda, Ciudad y Territorio, Por el cual se establecen las responsabilidades de los beneficiarios del subsidio familiar de vivienda urbana en especie, se establecen las condiciones para su restitución y se dictan otras disposiciones, Bogota: Presidencia de la República de Colombia; 2013.

[13] Mesa V. Universidad de Los Andes, La Vivienda de Interés Social, Principios y Retos, Bogota: Revista de Ingeniería, Uniandes, 2012. 